ISSN 2306-1561

Automation and Control in Technical Systems (ACTS)

2014, No 1.1(8), pp. 25-34.

DOI: $10.12731 / 2306-1561-2014-1-3$

\title{
Multi criteria decision making methodology for multimodal delivery of fast moving consumer goods
}

\section{Gavrikova Juliya Anatol'evna}

Russian Federation, Postgraduate Student, Department of «Road transport».

Moscow Automobile \& Road construction State Technical University, 125319, Russian Federation, Moscow, Leningradsky prospekt, 64. Tel.: +7 (499) 151-64-12. http://www.madi.ru

ulia.ovsyanikova@gmail.com

\begin{abstract}
In the article the multi criteria decision making model for multimodal delivery description is given. Methodology of the multi criteria decision making model for multimodal delivery of fast moving consumer goods is presented. Main areas of model and methodology application in fast moving consumer goods are provided.
\end{abstract}

Keywords: methodology, model, fast moving consumer goods, multi criteria decisionmaking, multimodal delivery. 
ISSN 2306-1561

Автоматизация и управление в технических системах (АУТС) 2014. - №1.1(8). - C. 25-34.

DOI: $10.12731 / 2306-1561-2014-1-3$

УДК 656.073

\section{Методика многокритериального выбора}

варианта мультимодальной доставки товаров

народного потребления

\section{Гаврикова Юлия Анатольевна}

Российская Федерация, аспирант кафедры «Автомобильные перевозки».

ФГБОУ ВПО «Московский автомобильно-дорожный государственный технический университет (МАДИ)», 125319, Российская Федерация, г. Москва, Ленинградский проспект, д.64, Тел.: +7 (499) 151-64-12, http://www.madi.ru

ulia.ovsyanikova@gmail.com

Аннотация. В статье приведено описание модели многокритериального выбора варианта мультимодальной доставки. Приведена методика многокритериального выбора варианта мультимодальной доставки. Приведены основные области применения модели и методики в компаниях-производителях товаров народного потребления.

Ключевые слова: методика, модель, товары народного потребления, методы решения мнококритериальных задач, мультимодальная доставка.

\section{1. Введение}

Сектор ТНП (от английского fast moving consumer goods (FMCG), что в переводе означает товары повседневного спроса, или товары народного потребления) - наиболее динамичный и быстро развивающийся сегмент мировой экономики. Тем не менее, это и один из самых жёстких, конкурентных секторов всех национальных и мировых экономик. Одновременно с этим сектор ТНП устойчив к экономическим кризисам, так как спрос на товары данной категории остаётся фактически неизменным в любые времена.

Сектор ТНП - рынок недорогих продуктов, которые быстро продаются и имеют недолгий срок службы (предметы личной гигиены, мыло, косметика, продукция для ухода за полостью рта, средства для бритья, бытовая химия, посуда, элементы питания, канцтовары). Иногда к данному сектору относят и фармацевтику, бытовую электронику и ряд другой продукции. Среди гигантов сектора ТНП - Japan Tobacco 
International, Philip Morris, British American Tobacco, Reckitt Benckiser, Colgate, Procter \& Gamble, Henkel, Unilever, Coca-Cola, PepsiCo, Nestle, Danone, Mars, Heinz, Kraft, Cadbury, Carlsberg, SUN Interbrew, Heineken, SABMiller-Anadolu Efes и другие.

Сектор ТНП динамичен. Поэтому, чтобы добиться лидерства и удержать его, компании также должны уметь быстро реагировать на изменения, гибко подстраиваться под новую конъюнктуру и принимать оперативные решения. Чтобы преуспеть в секторе ТНП, компании-производителю необходимо иметь представление о товаре и ценах на рынке, предоставить потребителю широкую продуктовую линейку, разработать грамотную стратегию мерчендайзинга и, конечно же, самое главное, без чего все остальное не имеет смысла, обеспечить доступность товара в правильное время и в правильном месте. Одним из потенциально перспективных направлений оптимизации в области планирования поставок ТНП от заводов до дистрибуционных центров является «умное» использование мультимодальных перевозок. Под «умными» мультимодальными перевозками подразумевается выбор оптимальных маршрутов мультимодальной доставки на основе моделей многокритериального выбора варианта доставки, адаптированных для сектора ТНП.

Результаты проведённого обзора российских и зарубежных исследований в диссертационной работе показали, что в настоящее время моделей, учитывающих специфику сектора ТНП, нет. Такие методы выбора варианта мультимодальной доставки как Electre, AHP и Promethee относятся к универсальным моделям, предлагающим общую методику без приспособления под определённую область применения. Для использования в секторе ТНП народного хозяйства, модели необходимо доработать под специфику данного сектора и вписать в существующие практики работы и учесть все особенности сектора. В частности, для данного сектора требуется расширить и конкретизировать номенклатуру параметров, учитываемых при расчётах, разработать методику расчётов и создать автоматизированную модель расчётов с применением метода решений многокритериальных задач и учитывающую все особенности сектора.

\section{2. Модель многокритериального выбора варианта мультимодальной доставки ТНП}

Целью разработки модели многокритериального выбора варианта доставки на примере ТНП является разработка научных и методологических основ многокритериального выбора варианта мультимодальной перевозки с учётом потребностей и особенностей российского сектора ТНП.

Модель многокритериального выбора варианта мультимодальной доставки ТНП, исходя из результатов анализа потребностей компаний-производителей ТНП, должна отвечать следующим требованиям [1 - 4]:

- учитывать при анализе параметры, важные для сектора;

- увеличить независимость решения;

- обеспечить простоту и лёгкость анализа; 
- обеспечить автоматизацию процессов;

- исключить ошибки расчёта;

- предоставлять возможность однозначного выбора оптимального маршрута;

- быть гибкой к изменениям логики анализа в случае возникновения потребности доработки;

- быть простой и удобной в использовании.

Согласно проведённому в диссертационной работе анализу исследований в области параметров, влияющих на выбор варианта доставки и опроса компанийпроизводителей ТНП, в модели многокритериального выбора варианта мультимодальной доставки учитываются следующие параметры - время доставки, стоимость доставки, гибкость доставки, риски доставки, влияние на уровень страховых запасов и влияние на окружающую среду. За основу модели взять метод Promethee [1].

Задача многокритериального выбора варианта мультимодальной доставки ТНП, учитывающая ранее описанные параметры (время доставки, стоимость доставки, риски, гибкость доставки, влияние на уровень страховых запасов, влияние на окружающую среду), может быть сформулирована следующим образом:

$$
\operatorname{Min}_{\mathrm{i}} \sum_{\mathrm{j}=1}^{6} \mathrm{w}_{\mathrm{j}} \mathrm{f}_{\mathrm{j}}\left(\mathrm{A}_{\mathrm{i}}\right)
$$

где $\mathrm{A}=\left\{\mathrm{A}_{1}, \mathrm{~A}_{2}, \ldots, \mathrm{A}_{\mathrm{i}}, \ldots \mathrm{A}_{\mathrm{n}}\right\}$ - множество альтернативных маршрутов;

$\mathrm{f}_{\mathrm{j}}\left(\mathrm{A}_{\mathrm{i}}\right)$ - значение критерия $\mathrm{j}$ для маршруту $\mathrm{A}_{\mathrm{i}}\left(\mathrm{f}_{1}-\right.$ время доставки $\mathrm{T}_{\text {дост }}, \mathrm{f}_{2}-$ стоимость доставки $\mathrm{C}_{\text {дост }}, \mathrm{f}_{3}$ - риски на маршруте $\mathrm{R}_{\text {марш }}, \mathrm{f}_{4}$ - гибкость доставки, $\mathrm{f}_{5}-$ влияние на уровень страховых запасов $\Delta \mathrm{SS}, \mathrm{f}_{6}$ - влияние на окружающую среду $\mathrm{E}_{\text {марш }}$;

$\mathrm{w}_{\mathrm{j}}$ - веса соответствующих критериев, указанных выше. Для $\mathrm{w}_{\mathrm{j}}$ должно выполняться следующее равенство:

$$
\sum_{j=1}^{6} w_{j}=1
$$

Каждое из $\mathrm{A}_{\mathrm{i}}$ представляет собой набор из десяти параметров, определённые ранее как вводные параметры для модели (страховой запас на исходном маршруте, вид транспорта, тип подвижного состава, расстояние, район следования, количество пересечений границы с РФ, количество пересечений границы с другими странами, количество водителей, количество перевалочных пунктов, тип перевалочного пункта). Без ограничения общности, маршруты будем обозначать $A_{i}, A_{k}$, где i, $k \in[1, n]$.

После того, как заданы вводные параметры для маршрутов, производится расчёт значений функций соответствующих критериев для каждого маршрута. Данные представлены в таблице 1. 


\section{Таблица 1 - Функции критериев на маршрутах}

\begin{tabular}{|c|c|c|c|c|c|}
\hline \multirow{2}{*}{ Маршрут } & \multicolumn{5}{|c|}{ Критерии } \\
\cline { 2 - 6 } & $\mathbf{f}_{\mathbf{1}}$ & $\mathbf{f}_{\mathbf{2}}$ & $\mathbf{f}_{\mathbf{3}}$ & $\cdots$ & $\mathbf{f}_{\mathbf{6}}$ \\
\hline $\mathrm{A}_{1}$ & $\mathrm{f}_{1}\left(\mathrm{~A}_{1}\right)$ & $\mathrm{f}_{2}\left(\mathrm{~A}_{1}\right)$ & $\mathrm{f}_{3}\left(\mathrm{~A}_{1}\right)$ & $\cdots$ & $\mathrm{f}_{6}\left(\mathrm{~A}_{1}\right)$ \\
\hline $\mathrm{A}_{2}$ & $\mathrm{f}_{1}\left(\mathrm{~A}_{2}\right)$ & $\mathrm{f}_{2}\left(\mathrm{~A}_{2}\right)$ & $\mathrm{f}_{3}\left(\mathrm{~A}_{2}\right)$ & $\cdots$ & $\mathrm{f}_{6}\left(\mathrm{~A}_{2}\right)$ \\
\hline$\vdots$ & $\vdots$ & $\vdots$ & $\vdots$ & $\vdots$ & $\vdots$ \\
\hline $\mathrm{A}_{\mathrm{i}}$ & $\mathrm{f}_{1}\left(\mathrm{~A}_{3}\right)$ & $\mathrm{f}_{2}\left(\mathrm{~A}_{3}\right)$ & $\mathrm{f}_{3}\left(\mathrm{~A}_{3}\right)$ & $\cdots$ & $\mathrm{f}_{6}\left(\mathrm{~A}_{3}\right)$ \\
\hline$\vdots$ & $\vdots$ & $\vdots$ & $\vdots$ & $\vdots$ & $\vdots$ \\
\hline $\mathrm{A}_{\mathrm{n}}$ & $\mathrm{f}_{1}\left(\mathrm{~A}_{\mathrm{n}}\right)$ & $\mathrm{f}_{2}\left(\mathrm{~A}_{\mathrm{n}}\right)$ & $\mathrm{f}_{3}\left(\mathrm{~A}_{\mathrm{n}}\right)$ & $\cdots$ & $\mathrm{f}_{6}\left(\mathrm{~A}_{\mathrm{n}}\right)$ \\
\hline
\end{tabular}

Структура предпочтений определяется посредством попарного сравнения маршрутов по каждому из критериев:

$$
d_{j}\left(A_{i}, A_{k}\right)=f_{j}\left(A_{i}\right)-f_{j}\left(A_{k}\right) \forall A_{i}, A_{k} \in A j=\overline{1,6}
$$

Будем говорить, что маршрут $A_{i}$ предпочтительнее маршрута $A_{k}\left(A_{i}>A_{k}\right)$ тогда и только тогда, когда:

$$
\left\{\begin{array}{c}
\forall \mathrm{J}: \mathrm{f}_{\mathrm{j}}\left(\mathrm{A}_{\mathrm{i}}\right) \leq \mathrm{f}_{\mathrm{j}}\left(\mathrm{A}_{\mathrm{k}}\right) \\
\exists \mathrm{k}: \mathrm{f}_{\mathrm{k}}\left(\mathrm{A}_{\mathrm{i}}\right)<\mathrm{f}_{\mathrm{k}}\left(\mathrm{A}_{\mathrm{k}}\right)
\end{array}\right.
$$

Также будем говорить, что маршрут $A_{i}$ равнозначен маршруту $A_{k}\left(A_{i}=A_{k}\right)$ тогда и только тогда, когда:

$$
\forall \mathrm{j}: \mathrm{f}_{\mathrm{j}}\left(\mathrm{A}_{\mathrm{i}}\right)=\mathrm{f}_{\mathrm{j}}\left(\mathrm{A}_{\mathrm{k}}\right)
$$

Для каждого критерия $\mathrm{j}(\mathrm{j}=\overline{1,6})$ также введём нормализованную (обобщённую) функцию предпочтений:

$$
\begin{aligned}
& P_{j}\left(A_{i}, A_{k}\right)=F_{j}\left[d_{j}\left(A_{i}, A_{k}\right)\right] \forall A_{i}, A_{k} \in A, \\
& \text { так что } \forall A_{i}, A_{k} \in A: 0 \leq F_{j}\left(A_{i}, A_{k}\right) \leq 1
\end{aligned}
$$

Данная функция показывает превосходство маршрута $A_{k}$ над маршрутом $A_{i}$ по критерию $\mathrm{f}_{\mathrm{j}}$ и обладает следующим свойством:

$$
P_{j}\left(A_{i}, A_{k}\right)>0=>P_{j}\left(A_{k}, A_{i}\right)=0,
$$


Существуют различные примеры обобщённых функций предпочтений. Рассмотрим функцию линейного предпочтения с областью безразличия, которая может быть выражена в виде:

$F(x)=\left\{\begin{array}{c}0, x \leq q \\ \frac{x-q}{p-q}, q<x \leq p, \\ 1, x>p\end{array}\right.$

где $\mathrm{x}$ - переменная;

$\mathrm{q}$ - порог безразличия;

$\mathrm{p}$ - порог строгого предпочтения.

Графическое отображение данной функции представлено на рисунке 1.

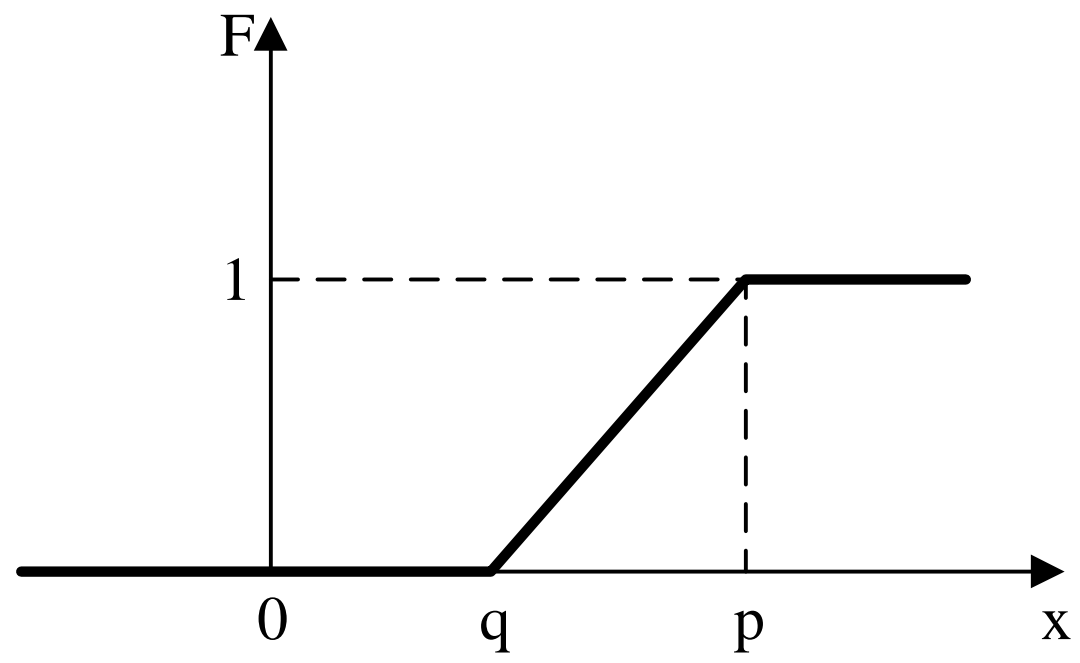

Рисунок 1 - Графическое отображение функции F(x)

Для решения задачи многокритериального выбора варианта мультимодальной доставки $\mathrm{q}_{\mathrm{j}}$ и $\mathrm{p}_{\mathrm{j}}$ будут определяться следующим образом:

$$
q_{j}=0, p_{j}=\max _{i} f_{j}\left(A_{i}\right)-\min _{i} f_{j}\left(A_{i}\right), j=\overline{1,6}, i=\overline{1, n}
$$

Введём понятие агрегированного индекса предпочтений:

$$
\pi\left(\mathrm{A}_{\mathrm{i}}, \mathrm{A}_{\mathrm{k}}\right)=\sum_{\mathrm{j}=1}^{6} \mathrm{P}_{\mathrm{j}}\left(\mathrm{A}_{\mathrm{i}}, \mathrm{A}_{\mathrm{k}}\right) \times \mathrm{w}_{\mathrm{j}} \forall \mathrm{A}_{\mathrm{i}}, \mathrm{A}_{\mathrm{k}} \in \mathrm{A}
$$

Функция $\pi\left(A_{i}, A_{k}\right)$ показывает, в какой степени маршрут $A_{i}$ менее предпочтителен, чем маршрут $A_{k}$ по всем критериям в совокупности.

Функция $\pi\left(A_{i}, A_{k}\right)$ обладает следующими свойствами:

$$
\left\{\begin{array}{c}
\pi\left(A_{i}, A_{i}\right)=0 \\
0 \leq \pi\left(A_{i}, A_{k}\right) \leq 1 \\
0 \leq \pi\left(A_{i}, A_{k}\right)+\pi\left(A_{k}, A_{i}\right) \leq 1
\end{array}\right.
$$


Каждый маршрут $A_{\mathrm{i}}$ сравнивается с $\mathrm{n}-1$ другими маршрутами.

Введём следующие функции:

ранг превосходства над маршрутом $A_{i}$

$\varphi^{+}\left(\mathrm{A}_{\mathrm{i}}\right)=\sum_{\mathrm{x} \in \mathrm{A}} \pi\left(\mathrm{A}_{\mathrm{i}}, \mathrm{x}\right)$

ранг превосходства маршрута $A_{i}$

$\varphi^{-}\left(\mathrm{A}_{\mathrm{i}}\right)=\sum_{\mathrm{x} \in \mathrm{A}} \pi\left(\mathrm{x}, \mathrm{A}_{\mathrm{i}}\right)$

Функция $\varphi^{+}\left(\mathrm{A}_{\mathrm{i}}\right)$ показывает, в какой степени маршрут $\mathrm{A}_{\mathrm{i}}$ хуже других маршрутов. Чем меньше $\varphi^{+}\left(A_{i}\right)$, тем лучше маршрут.

Функция $\varphi^{-}\left(A_{i}\right)$ показывает, в какой степени маршрут $A_{i}$ лучше других маршрутов. Чем больше $\varphi^{-}\left(\mathrm{A}_{\mathrm{i}}\right)$, тем лучше маршрут.

Введём функцию:

$\varphi\left(\mathrm{A}_{\mathrm{i}}\right)=\varphi^{+}\left(\mathrm{A}_{\mathrm{i}}\right)-\varphi^{-}\left(\mathrm{A}_{\mathrm{i}}\right)$

Чем меньше значение $\varphi\left(A_{i}\right)$, тем лучше маршрут $A_{i}$.

Причём функция $\varphi$ обладает следующими свойствами:

$$
\begin{aligned}
& -1 \leq \varphi\left(A_{i}\right) \leq 1 \\
& \sum_{A_{i} \in A} \varphi\left(A_{i}\right)=0
\end{aligned}
$$

Таким образом:

$A_{i}>A_{k}$ (маршрут $A_{i}$ предпочтительнее маршрута $A_{k}$, если $\varphi\left(A_{i}\right)<\varphi\left(A_{k}\right) . A_{i}=$ $\mathrm{A}_{\mathrm{k}}$ (маршрут $\mathrm{A}_{\mathrm{i}}$ равнозначен маршруту $\left.\mathrm{A}_{\mathrm{k}}\right)$, если $\varphi\left(\mathrm{A}_{\mathrm{i}}\right)=\varphi\left(\mathrm{A}_{\mathrm{k}}\right)$.

\section{3. Методика многокритериального выбора варианта мультимодальной доставки ТНП}

Алгоритм методики представляет собой комбинацию вычисления критериев на основе вводных данных по маршрутам и сравнения результатов с использованием метода решения многокритериальных задач Promethee. Детальный алгоритм представлен на рисунке 2.

На первом этапе определяется вес каждого критерия для компании-производителя ТНП с учётом её текущих приоритетов и целей.

На последнем этапе алгоритма методики, если метод решения многокритериальных задач не дал однозначного оптимального маршрута, требуется выбрать из отобранных альтернатив маршрут по критерию с наибольшим весом, указанном при вводе данных лицом, принимающим решение. 


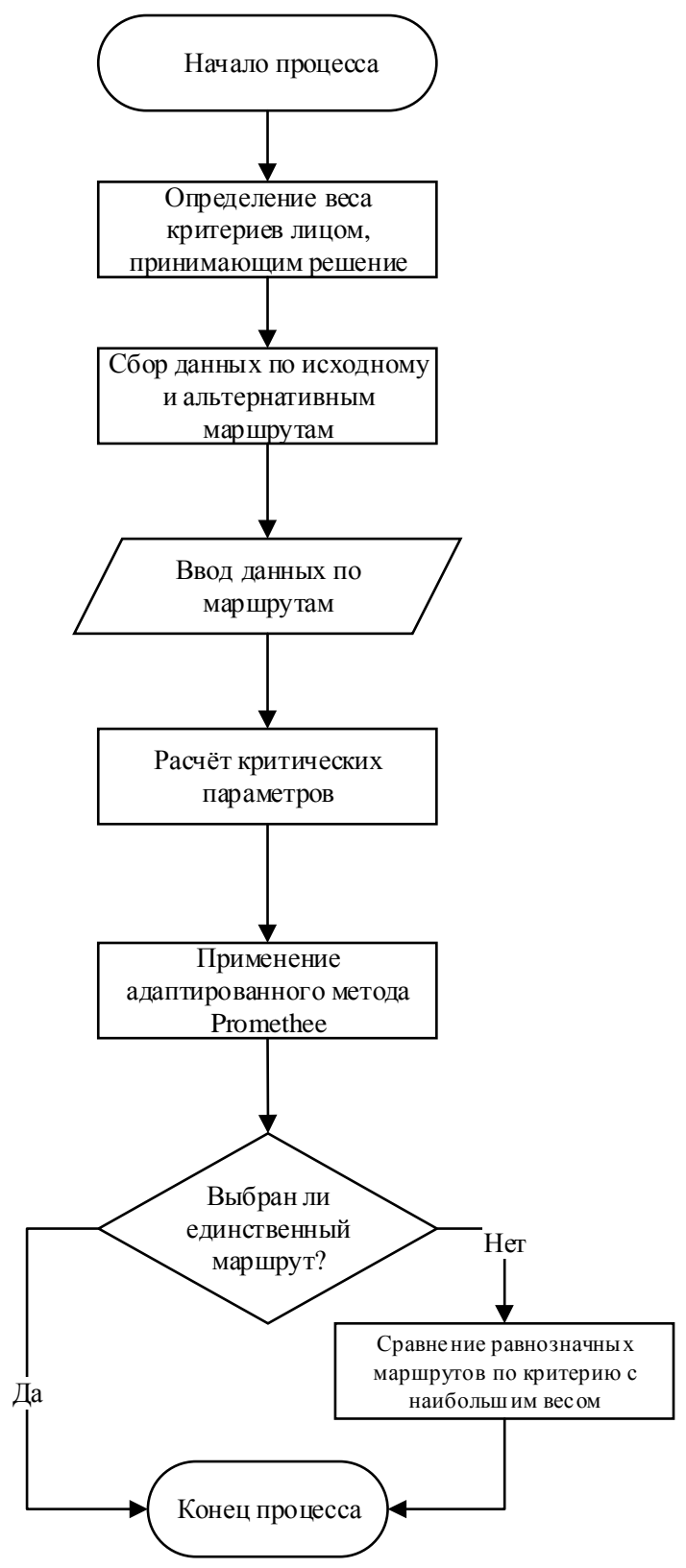

Рисунок 2 - Алгоритм методики многокритериального выбора варианта мультимодальной доставки ТНП

Высокоуровневая методика многокритериального выбора варианта мультимодальной доставки ТНП имеет следующую структуру:

1) Определение веса критериев лицом, принимающим решение;

2) Сбор данных по исходному и альтернативным маршрутам;

3) Ввод данных в модель;

4) Расчёт параметров;

5) Применение адаптированного метода Promethee;

6) Принятие решения по выбору маршрута.

Далее рассмотрим каждый пункт более детально.

1. Определение веса критериев лицом, принимающим решение 
Лицу, принимающему решение, требуется проранжировать параметры (стоимость доставки, время доставки, риски доставки, гибкость, влияние на окружающую среду и на уровень страховых запасов) в соответствие с текущими приоритетами и целями компании-производителя ТНП. Ранжирование параметров производится от 0 до 1 , где 1 - это максимальная важность, а 0 - минимальная.

2. Сбор данных по исходному и альтернативным маршрутам.

На данном этапе проводятся следующие действия:

- Определить исходный маршрут для анализа;

- На основе исходного маршрута выбрать маршруты для сравнения;

- Каждый маршрут разбить на этапы согласно следующим ограничениям:

- Этап содержит только один вид транспорта;

- Этапу соответствует один вид транспортной единицы;

- Собрать данные по каждому этапу согласно обязательному списку вводных параметров.

3. Ввод данных по маршрутам является основополагающим для получения корректных рекомендаций по выбору способа доставки. Для каждого этапа маршрута требуется внести вводные данные согласно установленным требования модели (с учетом количества знаков после запятой, фиксированных значений и др.).

4. Расчёт параметров производится согласно установленным формулам, логикам и вспомогательной базы данных, описанных в предыдущем параграфе.

Особенности расчёта параметров включают в себя:

- После заполнения данных необходимо нажать кнопку «Обновить расчёты» для проведения автоматических расчётов;

- Расчёты по всем установленным показателям производятся как для каждого этапа, так и в целом по маршруту;

- Если при проведении расчётов возникнет ошибка в каком-либо поле, то в нем будет высвечена надпись Error.

5. Применение адаптированного метода Promethee является последним шагом перед принятием решения о выборе оптимального маршрута доставки при заданных условиях. Данный процесс проходит в несколько этапов:

- После завершения всех расчётов и удостоверения, что нет ошибок в расчётах, требуется перейти на вкладку Promethee и нажать кнопку «Обновить расчёты»;

- По завершении расчётов будет построена таблица с итоговыми рейтингами по маршрутам. Маршрут с наивысшим рейтингом является оптимальным с точки зрения стоимости, времени доставки, рисков, гибкости, влияния на окружающую среду и страховые запасы;

- Если оценка с помощью модели не дала однозначного решения, то требуется провести дополнительное сравнение маршрутов с одинаковым рейтингом. Сравнение вариантов доставки проводится по критерию, который был определён лицом, принимающим решение, как наиболее важный для 
компании-производителя ТНП в текущей момент времени и текущей рыночной ситуации.

6. Принятие окончательного решения об использовании маршрута, рекомендованного моделью, в практической деятельности, является прерогативой лица, принимающего решение. Предложенная модель лишь помогает сравнению маршрутов и идентификации оптимального маршрута по заданным параметрам, учитывающим особенности и потребности компаний-производителей ТНП.

\section{4. Заключение}

Применение разработанной модели и методики в ТНП секторе возможно как компаниями-производителями ТНП, так и транспортными и логистическими компаниями. Для полного использования функционала модели и методики рекомендуется использовать для планирования доставки с заводов до дистрибуционных центров в международном сообщении. На основе проведённой оценки было выявлено, что применение разработанной модели и методики в компаниях-производителях ТНП позволяет достичь:

- улучшения эффективности использования мультимодального транспорта на маршруте до $+15 \%$;

- уменьшения затрат на доставку до $15 \%$;

- уменьшения времени на анализ маршрутов и принятие решения на 25\%;

- уменьшения страховых запасов на 5\%

- увеличения правильности принятия решений;

- увеличения скорости и эффективности принятия решений;

- увеличения независимости принятия решений;

- оптимизации доставки товаров с точки зрения неколичественных критических параметров, таких как риски и гибкость.

\section{Список информационных источников}

[1] Bierwirth, C. On Transport Service Selection in Intermodal Rail/Road Distribution Networks / C. Bierwirth, T. Kirschstein, F. Meisel // BuR Business Research Official Open Access Journal of VHB. - 2012. - Vol. 5, No. 2. - pp. 198-219.

[2] Кузнецов И.А. Особенности реализации автоматизированной информационноаналитической системы центра планирования перевозок строительных грузов / А.В. Остроух, И.А. Кузнецов // Вестник МАДИ(ГТУ). - М.: МАДИ (ГТУ), 2008. Вып. 1(12). - С. 92-96.

[3] Куфтинова Н.Г. Процессно-ориентированный подход к автоматизации планирования и управления транспортировкой продукции предприятий промышленности / А.В. Остроух, Н.Г. Куфтинова // Вестник МАДИ - 2010. - Вып. 4(23). - C. 62-66.

[4] Ostroukh A.V., Kuftinova N.G. Automation of Planning and Management of the Transportation of Production for Food Processing Industry Enterprises // Automatic Control and Computer Sciences. - 2012. - Vol. 46. - No. 1. - pp. 41 - 48. DOI: 10.3103/S0146411612010063. 